\title{
Robust estimation of Ackerman angles for front-axle steered vehicles
}

\author{
Verónica Sáiz-Rubio, Francisco Rovira-Más, I shani Chatterjee, J osé María Molina Hidalgo \\ Department of Agricultural Engineering, Universidad Politécnica de Valencia, Spain
}

Correspondence: Francisco Rovira-Más. Address: Department of Agricultural Engineering, Universidad Politécnica de Valencia, 46022 Valencia, Spain. Telephone: 34-96-387-7291. E-mail: frovira@dmta.upv.es.

Received: J une 28, 2012

DOI : 10.5430/air.v2n2p18

Accepted: September 26, 2012

URL: http://dx.doi.org/10.5430/air.v2n2p18

\section{Abstract}

The multiple benefits of automating steering in agricultural vehicles have resulted in various autoguidance systems commercially available, most of them relying on satellite-based positioning. However, the fact that farm equipment is typically oversized, heavy, and highly powered poses serious challenges to automation in terms of safety and reliability. The objective of this research is to improve the reliability of front-wheel feedback signals as a preliminary stage in the development of stable steering control systems. To do so, the angle turned by each front wheel of a conventional tractor was independently measured by an optical encoder and fused to generate the Ackerman feedback angle. The proposed fusion algorithm analyzes the consistency of each signal with time and checks the coherence between left and right front wheels according to the vehicle steering mechanism. Field experiments demonstrated the benefits of using redundant sensors coupled through logic algorithms for estimating Ackerman angles as the harsh conditions of off-road environments often resulted in the unreliable performance of electronic devices.

\section{Key words}

Intelligent vehicles, Agricultural robotics, Sensor fusion, Wheel encoders, Auto-steering

\section{I ntroduction}

Driving a tractor in the field can be especially hard and tedious due to the repetitive nature of agricultural operations. Harvesting, spraying, fertilizing, and planting are common tasks that require full attention of farm vehicles operators for long periods of time, usually leading to physical and mental fatigue in drivers. In addition to the benefits of avoiding stressful attention-demanding driving between rows of plants or trees, intelligent vehicles (i. e. vehicles endowed with artificial intelligence techniques) with automated steering allow operators concentrate on alternative tasks and vehicle functions. In general, driving assistance improves efficiency in the use of machinery when overlaps are reduced or eliminated, and it can also increase operation safety and performance of inexperienced drivers. However, the automation of agricultural equipment also induces the occurrence of serious risks that need to be taken into account. Machines that typically are very heavy, oversized, and run by powerful diesel engines must never operate without strict safety rules. The principal parameter providing feedback to the actuation of a front-axle guided vehicle is the measurement of the actual angle that the front wheels turn after executing computer-generated commands, as the vehicle patented by Mailer ${ }^{[1]}$, which implemented a PID controller getting feedback information from a wheel-angle sensor. These traditional controllers have 
been successful for driving assistance in the field; even when the four wheels of agricultural platforms are individually driven and steered, the control inputs for the steering motors have been computed using PID control laws ${ }^{[2]}$. When accurate steering is necessary, the traditional loop may be expanded as the double closed-loop PID control law proposed by Xiaopeng et al. ${ }^{[3]}$ to reduce steering overshoots and keep errors below $1^{\circ}$. In this approach, the wheel steering angle was used as the feedback for the inner loop, and the angular velocity of the wheel as the feedback for the outer loop. The advent of steer-by-wire systems allows the application of variable steering ratios. The steering ratio is the quotient between the steering wheel angle and the front wheel angle, and it is fixed for traditional steering systems. Jiang-Yun et al. ${ }^{[4]}$ brought an alternative to traditional control methods based on feedback by implementing a steer-by-wire system in which the front wheel angle is controlled directly with the steering ratio. This option resulted in a simpler design. Regardless of the control law implemented, the actual response of the front wheels will be the major responsible for the vehicle position. Therefore, the real-time determination of the true angle turned by the steering wheels is a matter of great importance. However, conventional auto-steering systems tend to measure the angle of one of both front wheels and use the simplified bicycle model to emulate the dynamics of motion. The approach presented in this paper takes into account the special features of Ackerman steering and independently measures the angle of each wheel, using the properties of this geometry, and the redundancy of wheel angles, to make final estimations of Ackerman angles more robust and immune to sensor failure.

The majority—perhaps the totality—of commercial autoguidance systems for agricultural vehicles rely on GPS positioning information, mainly after the suppression of GPS selective availability in 2000 by the US Department of Defense. The accessibility to global localization favored the development of Precision Agriculture (PA) techniques and the application of automation technology to agricultural production. Nevertheless, GPS cannot provide the level of accuracy and renewal obtained with local perception sensors such as digital cameras and laser rangefinders. Small local adjustments or the presence of unexpected obstacles has proved the beneficial use of multiple guidance sensors and fusion techniques the solution with highest potential for off-road vehicle automation ${ }^{[5]}$. Such sensor combinations as GPS and machine vision through the Kalman filter ${ }^{[6]}$ and fuzzy logic ${ }^{[7]}$, or 3D scanning lasers ${ }^{[8]}$ have enhanced the performance of satellite navigation. Even the implementation of a video camera, a ladar, and an inertial measurement unit in a vehicle has been sufficient to achieve autonomous turnings in the headlands of citrus groves without the assistance of global positioning ${ }^{[9]}$. In any case, with or without GPS, automatic steering requires the design and implementation of a closed-loop control system, where the system to be controlled is often a hydraulic steering mechanism and the feedback signal is typically the front wheels turning angle. Given that fluid power actuators tend to behave nonlinearly, their characterization and control is usually intricate and complex ${ }^{[3,10]}$, what has led to various detailed studies ${ }^{[11-13]}$. However, much less attention has been devoted to the generation of reliable feedback signals, in spite of their crucial role within the control loop. Most of the conventional off-road vehicles used in agriculture incorporate Ackerman steering, either front-axle or inverse rear axle, which traditionally has been modeled following the bicycle model. A preliminary step towards navigational excellence starts with the optimization of the steering trapezium ${ }^{[14]}$, but the real-time estimation of the angle turned by the front wheels is normally the essential parameter to determine. This measurement has been traditionally carried out with flowmeters, linear potentiometers, and optical encoders, although the latter alternative offers the advantage of the direct measurement of the angle turned by the wheel ${ }^{[15]}$. Current architectures tend to incorporate encoders and potentiometers only in one of the front wheels, where no redundancy for the angle estimation is possible, as the system developed by Dickson et al. ${ }^{[16]}$ in which a rotary potentiometer aligned with the king pin of one of the front wheels was used to estimate the angles turned by a vehicle. Furthermore, although the bicycle model only considers one steering angle, denominated Ackerman angle, the actual vehicles have two front wheels which turn different angles according to the nonlinear law established by the linkage geometry. The objective of this investigation is to increase reliability and robustness in the estimation of the angles turned by front-axle off-road vehicles, with the final overall target of enhancing the stability of auto-steered vehicles. Field tests under different conditions have demonstrated that electronic devices such as optical encoders are subjected to harsh ambient conditions which many times result in sensor failures. Redundancy and signal conditioning at the feedback level offers a means to cope with sensor malfunctioning and, consequently, leads to strengthen the control of vehicles with automated guidance. 


\section{General architecture for sensing and controlling front-axle steering}

The vehicle used in the experiments is a conventional tractor (JD 5820, Deere \& Co., Moline, IL, USA) equipped with a turbocharged diesel engine of $90 \mathrm{HP}$ and hydrostatic steering. The tractor has been robotized with three types of sensors: localization, perception and control. Global positioning is available with a GPS receiver installed in the cabin of the tractor, and different digital cameras have been implemented in the vehicle to provide visual information of the tractor's surroundings for alternative applications based on machine vision. The angles turned by the left and right front wheels of the tractor were the principal measurements tracked in this research, and were carried out with two optical encoders (Bourns, Riverside, CA, USA) installed in the kingpin of each front wheel as illustrated in Figure 1. The encoders provide a resolution of 256 steps to cover the $360^{\circ}$ of the internal disk.

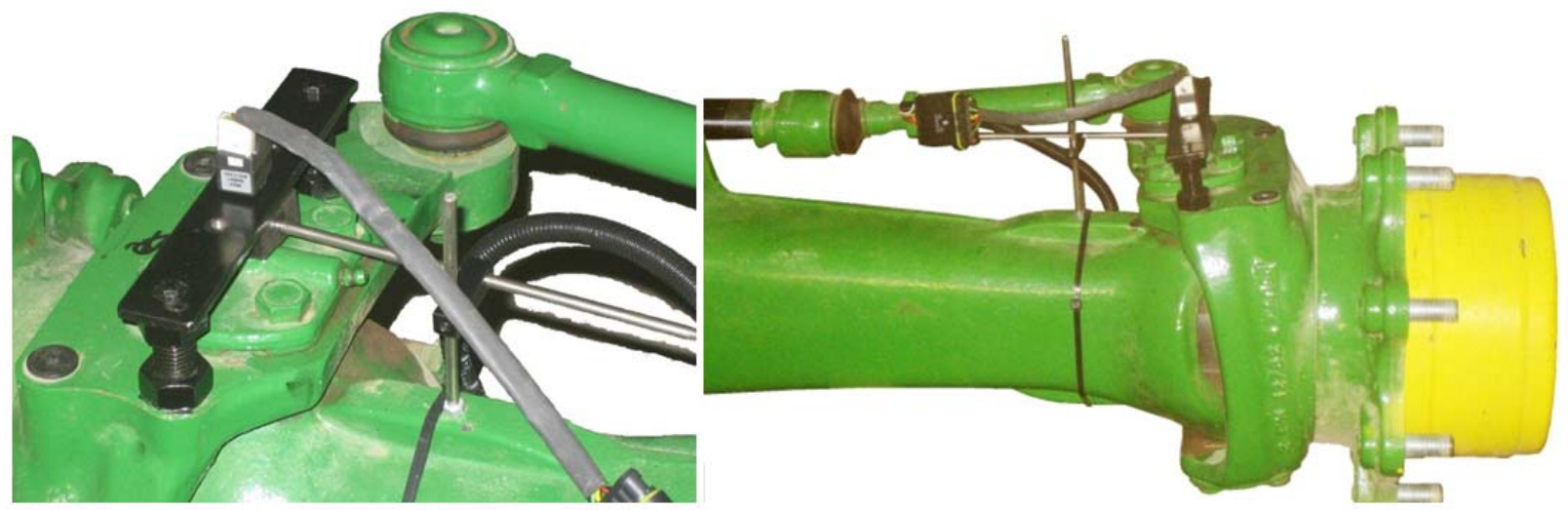

Figure 1. Installation of optical encoders in the kingpin of the front wheels

Given that the experimental vehicle uses front-axle steering, the angle turned by the left wheel is slightly different from that turned by the right wheel. The relationship between the turns executed by the front wheels is not linear and depends on the particular structure of the steering mechanism implemented in the vehicle. However, for small angles (under $10^{\circ}$ ) this relationship can be considered linear and vehicles with guided assistance often use a single feedback sensor to find the angle turned by one of the two wheels, since it is not difficult to calculate the other angle when the relationship between the rotations of both wheels is well known. To record the measurement of the angles with the computer onboard, the output of the encoders - a digital signal in the range 0 - $5 \mathrm{~V}$ - was acquired with a data acquisition board (NI-DAQmx, National Instruments, Austin, TX, USA) and input to the computer through one of its USB ports. Both encoders provided a direct measurement of the angle turned by the front wheels at a frequency of $10 \mathrm{~Hz}$. Because the encoder shaft was mechanically attached to the kingpin of each front wheel, the number of cycles counted by each encoder was proportional to the angle rotated by the corresponding wheel. The encoders were powered by a secondary $12 \mathrm{~V}$ battery that runs independently from the main battery of the vehicle. However, both batteries are charged by the tractor's alternator and mutually connected to provide extra power if a peak demand occurs. The auxiliary battery allowed starting and halting the engine without interrupting the power supply to sensors and computers. As the encoders are powered by a direct current of $5 \mathrm{~V}$, a voltage reducer was inserted in the electric circuitry. The angles output by each encoder were visualized and recorded through a customized C++ application whose graphic user interface is portrayed in Figure 2. This application allowed the recording of left and right wheel angles, the calculated Ackerman angle, the globally-referenced trajectory followed by the vehicle in East-North coordinates (Local Tangent Plane Coordinate System), and the heading and forward velocity of the tractor. The instantaneous acquisition of heading, speed, and position for every angle recorded is key to understand the behavior of the vehicle as well as the relationship between the angle turned by the front wheels and the resulting course traced by the vehicle for a given velocity. 


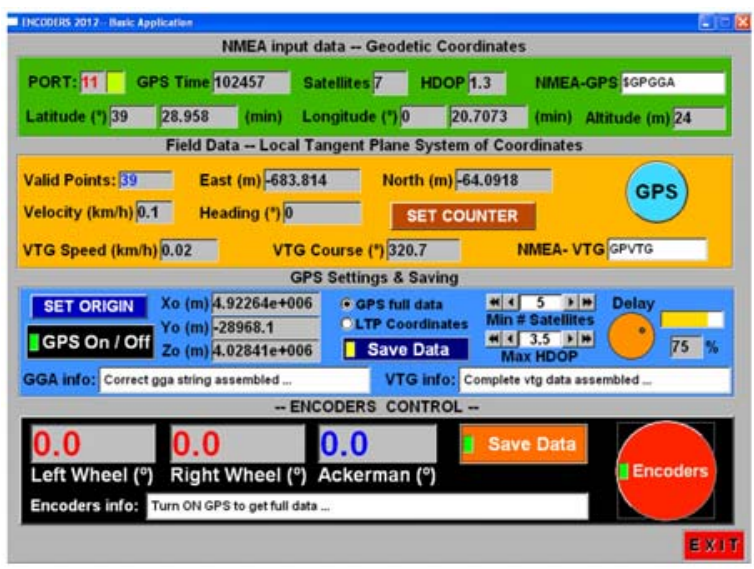

Figure 2. Customized interface to record essential data

The relationship between the angles turned by the left and right wheels could be directly deduced from the bar linkage of the tractor, but the accessibility to the steering mechanisms is not always favorable, and consequently it results more convenient to carry out a calibration test. The calibration test consists of turning the wheels slowly to cover the entire range of values from full lock to the left (counterclockwise) to the maximum turn to the right (clockwise), acquiring as many intermediate angles as necessary. In the calibration test performed, the actual angle rotated by each front wheel was estimated with a heavy-duty goniometer with a circular scale placed under each wheel. The angles measured by the encoders and displayed in the screen of Figure 2 could, therefore, be compared to the actual angles directly measured with the goniometer. The availability of the angles turned by each tire at different positions of the steering wheel allowed for the calculation of a fit equation relating left and right turns. This equation has been normally simplified with a straight line, but the quadratic fit of Figure 3 shows the benefits of using a second order equation to relate left and right turns. Note the excellent adjustment of the trend curve with the actual data for extreme angles (over $30^{\circ}$ ), as these severe turns have typically been the origin of the largest deviations between prediction and measurement when linear regression is used. While not common for traversing crop rows, which usually run straight and parallel to each other, sharp turns are necessary over the headlands at the end of the rows, where vehicles need to change rows and negotiate turns of $180^{\circ}$. A quantitative definition of what can be considered a turn taken by a vehicle can be extracted from the methodology patented by Lindores ${ }^{[17]}$, according to which a turn is a $30^{\circ}$ change in heading over a distance of $100 \mathrm{~m}$. The quadratic fit that best related left and right angles for the tractor front wheels is depicted in Figure 3, and its mathematical expression is given in Eq. 1, where $\mathrm{R}(\mathrm{k})$ represents the angle turned by the right wheel and $\mathrm{L}(\mathrm{k})$ is the left wheel angle, both expressed in degrees.

$$
\mathrm{L}(\mathrm{k})=-0.0034 \cdot \mathrm{R}^{2}(\mathrm{k})+0.9976 \cdot \mathrm{R}(\mathrm{k})+0.2587 ; \mathrm{r}^{2}=0.9998
$$

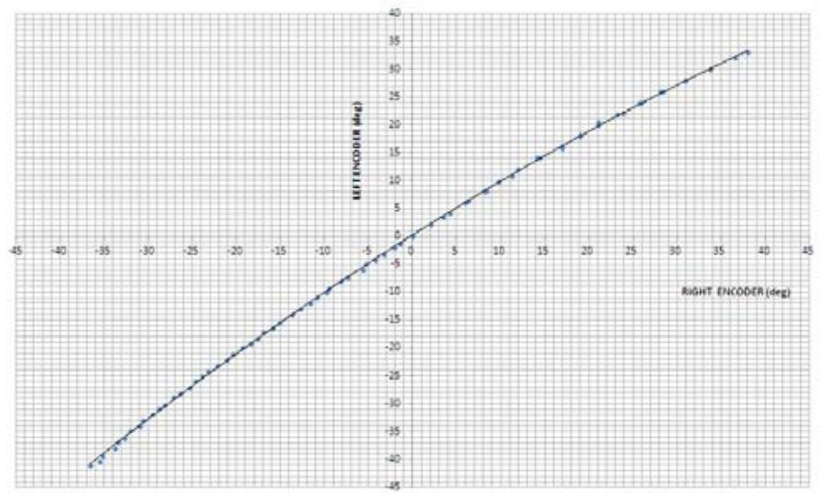

Figure 3. Quadratic equation that relates left and right wheel angles of the experimental tractor 


\section{Philosophy for the robust estimation of Ackerman angles}

The objective of the data fusion algorithm is to determine the Ackerman angle that best represents the turning condition of a vehicle to model path-planning algorithms and auto-steering control loops. The accomplishment of this goal started by analyzing and comparing the consistency of the angles measured with each of the encoders simultaneously. Once the angle measured by one of the encoders is known, the other can be predicted by applying Eq. 1. In particular, the angle turned by the left wheel was directly measured with the left encoder and also predicted from the angle output by the right encoder after the application of the regression equation given in Eq. 1. If $\mathrm{L}(\mathrm{k})$ represents the angle measured by the left encoder at sample point $\mathrm{k}$ and $\mathrm{R}(\mathrm{k})$ is the corresponding angle recorded by the right encoder at the same point $\mathrm{k}$, the principal measurement $x(k)$ is defined coincident with $L(k)$, that is, $x(k)=L(k)$, but its prediction $z(k)$ is calculated from the outputs of the right encoder after applying the regression equation of Fig. 3, as specified in Eq. 2.

$$
z(k)=-0.0034 \cdot R^{2}(k)+0.9976 \cdot R(k)+0.2587
$$

The analysis of consistency and further validation of the information acquired by the encoders was conducted in real time according to two fundamental concepts: integrity and continuity. There is integrity for a given time step k if measurement $\mathrm{x}(\mathrm{k})$ and prediction $\mathrm{z}(\mathrm{k})$ do not differ, in absolute value, more than a predefined threshold. This threshold was considered $7^{\circ}$ in the experiments conducted in the field and described in the following section; so, for instance, if $|x(345)-z(345)|<7^{\circ}$, there was integrity for point 345. Continuity, on the contrary, affects to each series of data independently in such a way that two consecutive angles, either from $\mathrm{R}(\mathrm{k})$ or $\mathrm{L}(\mathrm{k})$ are considered continuous only if their absolute difference remains below a certain threshold, which in this particular project varied from $3^{\circ}$ to $12^{\circ}$. The continuity of angles was conceived as a Boolean variable taking a value of one when there was continuity and zero otherwise. The lack of continuity in one encoder indicated a jump in the data series, usually caused by noise or a physical problem in the encoder. The calculation of the Ackerman angle is usually defined as the average of left and right wheel angles, but spurious angles, such as those induced by a deficient continuity or integrity, invalidate that simple calculation. For such cases, alternative equations must be applied in the calculation of Ackerman angles, keeping the most reliable information and discarding doubtful data. The application of the bicycle model to vehicles with frontal Ackerman geometry — front axle steering through a mechanical linkage that approaches perfect turns by making both wheels turn a different angle — necessarily leads to the combination of left and right angles into a theoretical turning angle that fits the dynamic (bicycle) model and eventually the implemented control system. When both angles are available, the Ackerman angle is typically their average.

Continuity may fail either for both encoders or, more likely, for just one of them for a certain value of k. Integrity, on the other hand, involves the data of both sensors. When there is no integrity and a jump is detected in the right encoder-i.e. there is no continuity for right angles although left angles are correct, the Ackerman angle A(k) can be estimated with Eq. 3 , where only data coming from the left encoder is taken into account, specifically the average of the current value and the previous one. Likewise, when continuity problems are found for the left encoder, $A(k)$ is the average of the current angle measured by the right encoder and the previous angle as given by Eq. 4 .

$$
\begin{aligned}
& \mathrm{A}(\mathrm{k})=(\mathrm{x}(\mathrm{k}-1)+\mathrm{x}(\mathrm{k})) / 2 \\
& \mathrm{~A}(\mathrm{k})=(\mathrm{R}(\mathrm{k}-1)+\mathrm{R}(\mathrm{k})) / 2
\end{aligned}
$$

When there exists integrity between measurement and prediction for value $\mathrm{k}$ but previous values of the series are not continuous in the last three angles (up to k-2), only current values (k) of both encoders are averaged as indicated by Eq. 5 . However, when there is integrity and continuity during the last three angles for both encoders, the smoothest value of $\mathrm{A}(\mathrm{k})$ is provided by the moving average of Eq. 6, which obviously is the optimal situation for a stable performance of steering controllers and navigation planners.

$$
\mathrm{A}(\mathrm{k})=(\mathrm{R}(\mathrm{k})+\mathrm{x}(\mathrm{k})) / 2
$$




$$
A(k)=(R(k)+x(k)+R(k-1)+x(k-1)+R(k-2)+x(k-2)) / 6
$$

The philosophy of the algorithm proposed is represented in Figure 4 as a block diagram. It is important to keep in mind that all the calculations and filtering checks are executed in real time, and therefore, 3-point moving matrices for every variable tracked need to be implemented in the code. Apart from left, right, and Ackerman wheel angles, integrity and continuity matrices were also recorded in text files for further analysis.

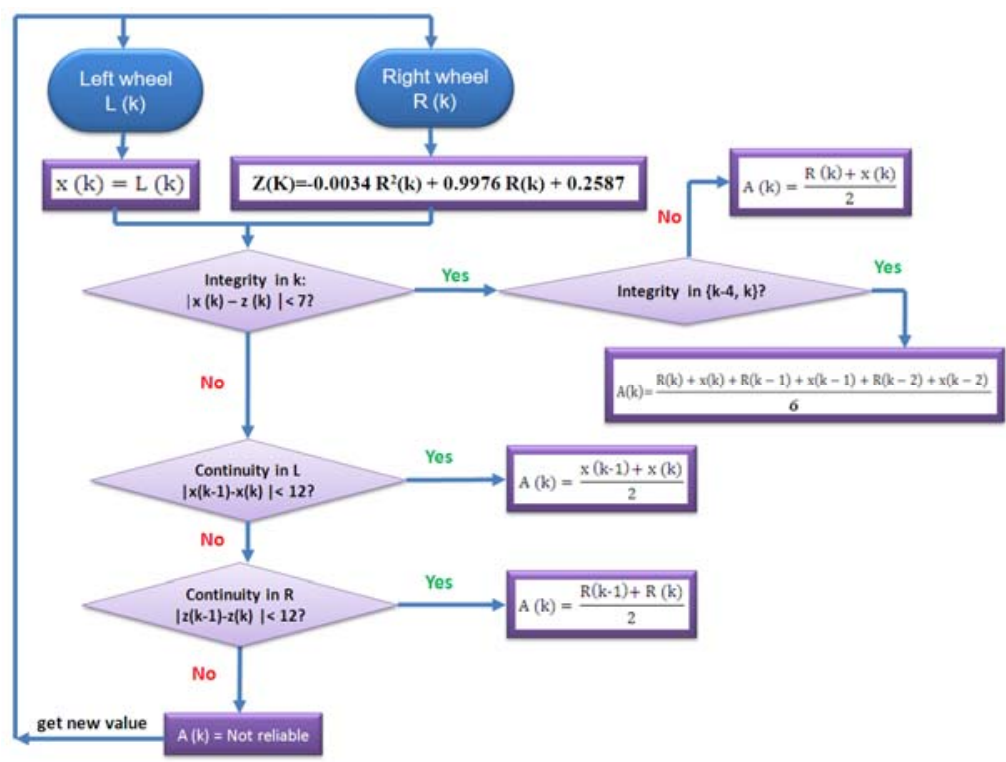

Figure 4. Flow chart of the algorithm proposed to enhance the calculation of Ackerman angles

\section{Field tests and experimental results}

The objective of the field tests was the general verification of the algorithm in real conditions found in citrus orchards and wine vineyards. Three different situations were detected in the field: one of the encoders suddenly stopped measuring as a consequence of mechanical or electrical failure (case 1), one of the encoders drifted over time (case 2), and both encoders behaved normally as expected (case 3). A detailed explanation for each case follows below.

\section{Case 1: Electromechanical failures in the wheel encoders}

The plots represented in Figure 5 show the angles turned by both front wheels measured by their respective encoders together with the Ackerman angle calculated with the algorithm outlined in Figure 4. The vehicle was driven through several rows of a citrus grove, turning in the headland at the end of each row as indicated by the large angles represented in the plot by peaks over $30^{\circ}$ followed and preceded by near-zero angular corrections. According to the top plot representing the steering maneuvers of the left wheel, the left encoder supplied reasonable values along the runs traveled in the orchard; however, the right encoder (central plot) shows a large jump short after point 14000 that invalidates the sensor readings after that value. The artifact created by the malfunctioning encoder was of such a great magnitude that the scale needed to represent its value flattened the peaks corresponding to the headland turns registered before the faulty point. The jump occurred around point 14000 fired the integrity filter that discarded the readings of the right encoder after the jump. As a result, the calculation of $A(k)$ averaged $x(k)$ and $R(k)$ before the jump as defined in Eq. 6, but only used the left angle values (Eq. 3) after the jump. Overall, the final Ackerman angle A(k) output by the algorithm during the entire test was not affected by unrealistic noisy estimates, as represented in the bottom graph of Figure 5, where the magnitude of the angles is bounded by $\pm 50^{\circ}$. 

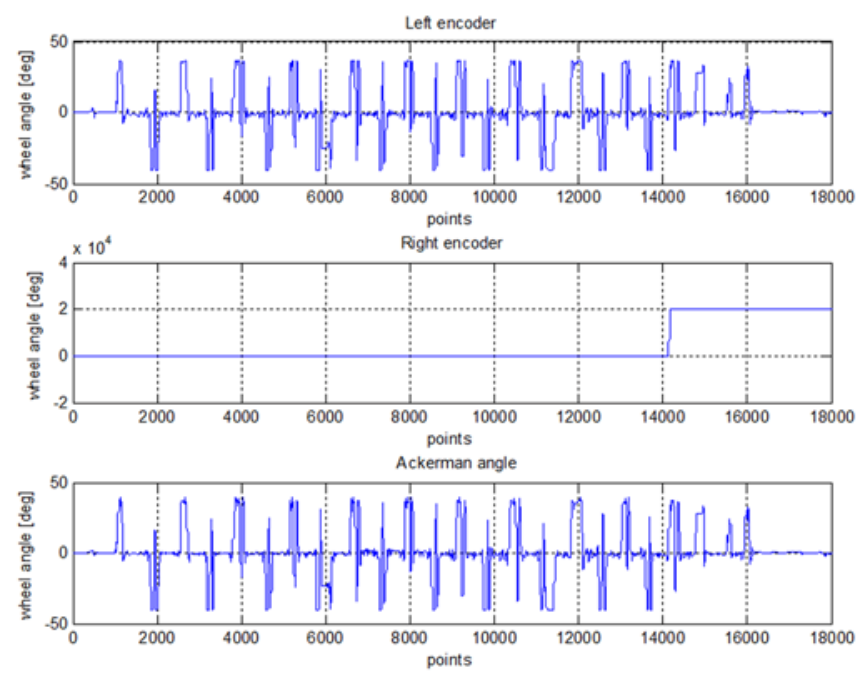

Figure 5. Ackerman angles with electromechanical failures in one of the encoders

\section{Case 2: Encoder drift}

This situation also involves a malfunction in one of the encoders, but unlike the previous case, the failure is more subtle this time; it occurs slowly, small when it appears but growing with time. The output for the left encoder (top plot) represented in Figure 6 indicates that the average angle, when the vehicle moves straight ahead, is approximately zero as expected. The right encoder (center plot) also shows the same trend at the beginning, but after point 6000 its average value starts to deviate from the logical zero degrees angle. In this situation, continuity is not helping to issue the initial warning because drift is a steady deviation that grows continuously. However, the values of integrity point at increasing differences between measured and predicted values that lead to discard drifting values after point 6000 , using thereafter only the estimates coming from the left encoder. As depicted in the bottom plot of Fig. 6, the final angle A(k) is not affected by the erroneous behavior of the right encoder, and the algorithm returns angles close to $0^{\circ}$ when the vehicle moves following straight rows. The origin of the drift in the right encoder was found long after the tests were carried out. It was caused by a current derivation in the wiring of the tractor cabin originated by the lack of ground pin in the computer plug. This problem was solved by using independent power sources so that the $5 \mathrm{~V}$ DC needed by the encoders was not affected by the power of computer and monitors aboard.
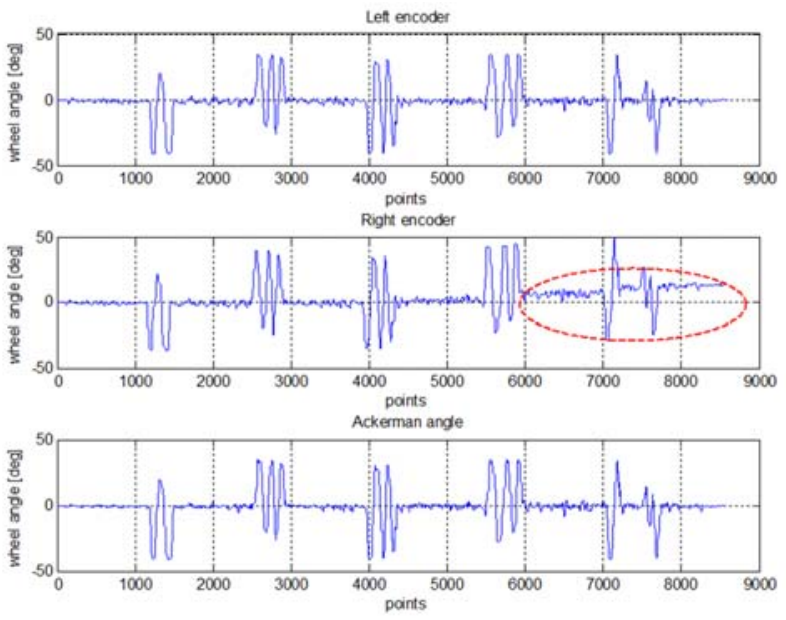

Figure 6. Ackerman angles with signal drift in one of the encoders 


\section{Case 3: Encoder redundancy}

When the information collected with the encoders is coherent, what happens most of the times, the task of the algorithm is to smooth the values of the final steering angle by implementing a three-point moving average. The three plots of Figure 7 are very similar among them, and the small differences noticed during large turns at the field headlands obey to the geometry of the steering linkage. The final output, depicted in the bottom plot of Figure 7 as the Ackerman angle, is stable and shows logical values for $\mathrm{A}(\mathrm{k})$. This stability is assured by the concepts of integrity and continuity. However, apart from filtering outliers and noisy estimates of the wheel angles, these concepts provide valuable information on the dynamics and behavior of the vehicle in its motion, especially when conducting automated tasks. The integrity plot of Fig. 8 demonstrates that the actuation of one of the wheels can be accurately predicted with the redundant measurement of the other; furthermore, deviations can be bounded by $6^{\circ}$, and in most of the cases are below $3^{\circ}$. The continuity values determined independently for each wheel, as depicted in Figure 9, show that variations in angles between consecutive points are insignificant when the vehicle follows straight rows but can reach $10^{\circ}$ in the $180^{\circ}$ turns needed to change consecutive rows. To fully understand these values, it is necessary to know the trajectory traced by the tractor, the distance between points, and the velocity reached by the vehicle at any point of its trajectory. Figure 10 provides the course followed by the vehicle among rows of a winery vineyard, and Figure 11 plots the instantaneous velocity at any point registered by the onboard computer.
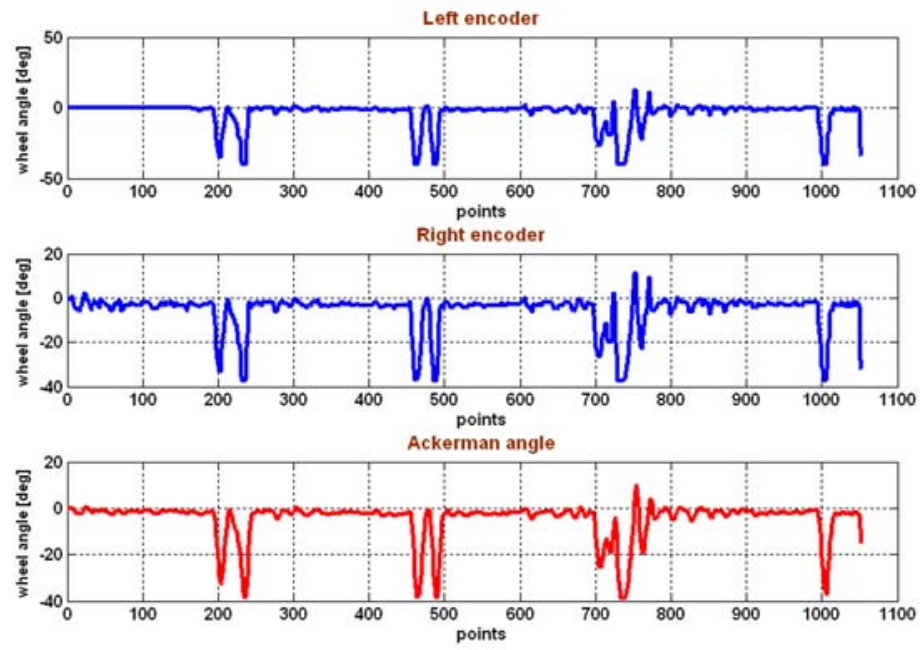

Figure 7. Left and right front-wheel angles and resulting Ackerman angle

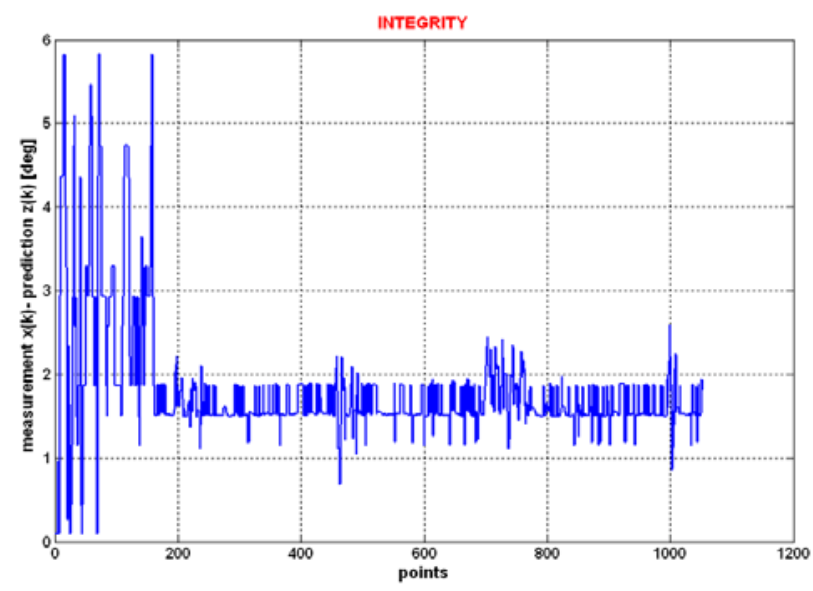

Figure 8. Integrity plot: quantitative differences between measurements and predictions 

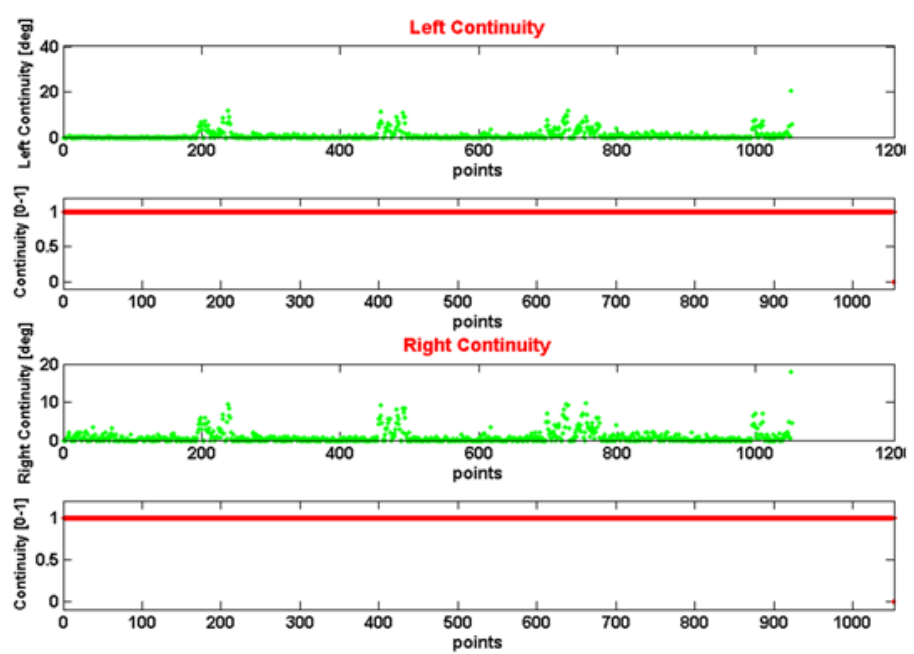

Figure 9. Continuity plots for left and right encoders

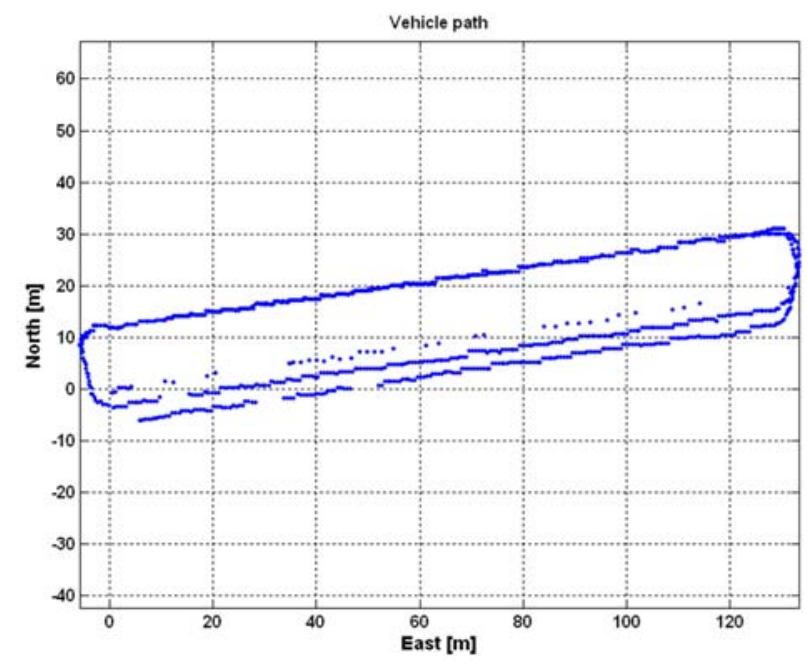

Figure 10. Trajectory traced by the vehicle along the vineyard rows

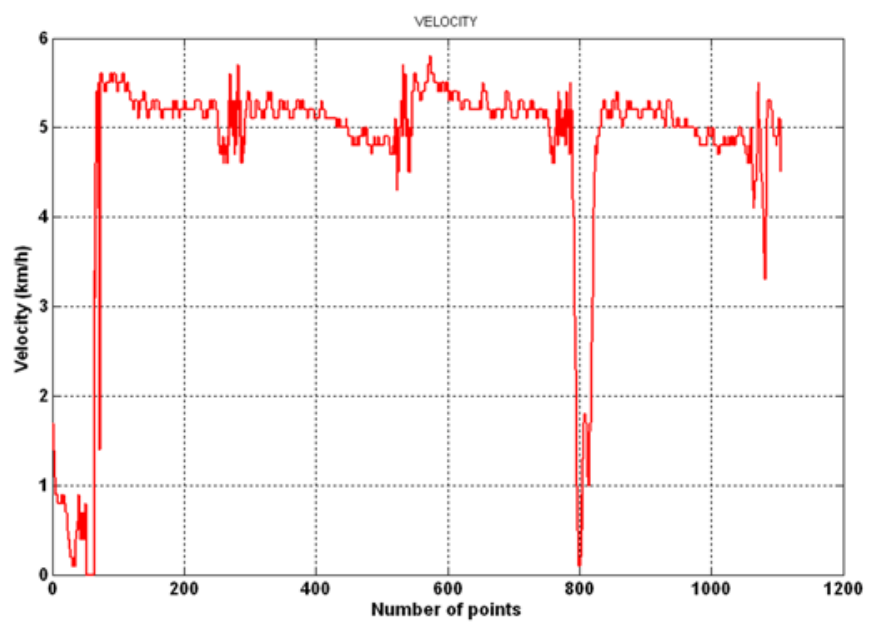

Figure 11. Forward velocity registered by the vehicle in its motion 


\section{Conclusions}

The high-level requirements of safety for automating off-road vehicles demand reliability for key components of the control system. The proper measurement of steering feedback signals is essential for achieving smooth autonomous navigation. The system proposed implements two optical encoders, one for each front wheel, and a fusion algorithm to enhance the estimation of the feedback angle. The presence of redundant sensors and the application of the fusion algorithm resulted in more stable angle measurements, as proved in field tests conducted in orchards and vineyards. Future upgrades of the algorithm will try to further elaborate on the concepts of continuity and integrity to get more solid responses during large turns at the end of rows. The ultimate stage in the development of this system will include its integration and evaluation in a complete auto-steered vehicle. The information retrieved from both encoders, the application of stability concepts such as integrity and continuity, and the availability of global positioning systems, when properly combined, allow the investigation of the correlation between the angles turned by the front wheels of an off-road vehicle and the final position reached by that vehicle when traveling at a certain speed, which still remains an unsolved problem in spite of its high interest for roboticists and engineers devoted to the automation of off-road vehicles.

\section{References}

[1] Mailer, R. L. 2005. Vehicle positioning apparatus and method. US Patent 6,876,920.

[2] Liu, C., Wang, M. \& Zhou, J. Coordinating control for an agricultural vehicle with individual wheel speeds and steering angles. Control Systems. 2008; 28(5): 21-24. http://dx.doi.org/10.1109/MCS.2008.927961

[3] Xiaopeng, W., Znoxi, Z., Long, Y., Zhigang, Z., Lian, H. \& Jian, H. Tractor steering control system design basing on double closed-loops control. Proc. of the IEEE Intern. Conf. on Intelligent Computation Technology and Automation. 2011 ; 2: $294-298$.

[4] Jiang-Yun, Y., Feng, K. \& Fang-Yuan, W. Control strategy for front wheel angle of steer-by-wire based on variable steering ratio. Proc. of the International Conference on Computer Science and Network Technology. 2011; 2: 852-856.

[5] Reid, J.F., Zhang, Q., Noguchi, N. \& Dickson, M. Agricultural automatic guidance research in North America. Computers and Electronics in Agriculture. 2000; 25 (2000): 155-167. http://dx.doi.org/10.1016/S0168-1699(99)00061-7

[6] Rovira-Más \& S. Han. Kalman filter for sensor fusion of GPS and machine vision. ASABE Pub. N 063034, St. Joseph, Mich. 2006.

[7] Rovira-Más, F., Han, S. \& Reid, J. F. Fuzzy logic model for sensor fusion of machine vision and GPS in autonomous navigation. ASABE Pub. No 051156, St. Joseph, Mich. 2005.

[8] Wang, L., Emura, T. \& Ushiwata, T. Automatic Guidance of a Vehicle Based on DGPS and 3D Map. Proc. of IEEE Conf. Intelligent Transportation Systems. Dearborn (MI), USA. 2000.

[9] Subramanian,V. \& Burks, T.F. Autonomous Vehicle Turning in the Headlands of Citrus Groves. ASAE Paper No. 071015 St. Joseph, Mich.: ASABE. 2007.

[10] Rovira-Más, F., Zhang, Q. \& Hansen, A. H. Dynamic behavior of an electrohydraulic valve: typology of characteristic curves. Mechatronics. 2007; 17: 551-561. http://dx.doi.org/10.1016/j.mechatronics.2007.07.003

[11] Zhang Q. Hydraulic linear actuator velocity control using a feedforward-plus-PID control. International Journal of Flexible Automation and Integrated Manufacturing. 1999; 7(3): 277-292.

[12] Zhang Q. A generic fuzzy electrohydraulic steering controller for off-road vehicles. Journal of Automobile Engineering. 2003; 217(9): 791-799. http://dx.doi.org/10.1177/095440700321700904

[13] Inoue, K. Steering control system for autonomous tractor. Proc. of the 7th JFPS International Symposium on Fluid Power, Toyama, Japan. 2008.

[14] Yao, K., Wang, Y., Hou, Z. \& Zhao, X. Optimum design and calculation of Ackerman Steering Trapezium. Proc. of the IEEE Intern. Conf. on Intelligent Computation Technology and Automation. 2008; 1: 1248-1252.

[15] Rovira-Más, F., Zhang, Q., Reid, J. F. \& Will, J. D. Machine vision based automated tractor guidance. International Journal of Smart Engineering System Design. 2003; 5: 467-480. http://dx.doi.org/10.1080/10255810390445300

[16] Dickson, M. A., Noguchi, N., Zhang, Q., Reid, J. F. \& Will, J. D. Sensor-fusion navigator for automated guidance of off-road vehicles. US Patent 6,445,983. 2002.

[17] Lindores, R. J. Method and system for determining the path of a mobile machine. US Patent 7,363,154. 2008. 